\title{
Linear Scleroderma
}

National Cancer Institute

\section{Source}

National Cancer Institute. Linear Scleroderma. NCI Thesaurus. Code C116780.

A type of localized scleroderma characterized by a long strip of indurated skin, which is typically found unilaterally on an arm or leg, and sometimes on the forehead or trunk. This disorder often affects the tissues beneath the skin, causing damage to bones, muscle or other organs. It can limit movement, alter growth, and disfigure the affected area. 